\section{Change in subfoveal choroidal thickness in central serous chorioretinopathy following spontaneous resolution and low-fluence photodynamic therapy}

NH Kang and YT Kim

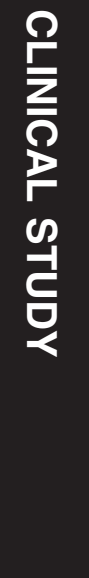

\begin{abstract}
Purpose To assess the change in subfoveal choroidal thickness (SFCT) in central serous chorioretinopathy (CSC) following spontaneous resolution and low-fluence photodynamic therapy (PDT) using the enhanced depth imaging optical coherence tomography (EDI-OCT).

Methods A total of 36 consecutive eyes of 36 patients were included in this retrospective study: 16 eyes with spontaneously resolved CSC and 20 eyes with PDT-treated CSC. Best-corrected visual acuity and SFCT were evaluated at each visit until complete absorption of the subretinal fluid. SFCT of 32 normal subjects were also measured, as the control group. Wilcoxon's singed-rank test was used to evaluate the effects of spontaneous resolution and PDT. To compare the SFCT of the eyes with resolved CSC with that of the normal eyes, Mann-Whitney $U$-test with Bonferroni correction was also employed.

Results SFCT of patients was $459.16 \pm 77.50 \mu \mathrm{m}$ at the baseline, and decreased to $419.31 \pm 54.49 \mu \mathrm{m}$ after a spontaneous resolution $(P=0.015)$. However, SFCT was not normalized in comparison with that of the normal subjects $(P<0.001)$. SFCT in PDT group was also reduced from $416.43 \pm 74.01$ to $349.50 \pm 88.99 \mu \mathrm{m}(P<0.001)$, with no significant difference with the normal value $(P=0.087)$.
\end{abstract}

Conclusions SFCT in patients with CSC decreased both after spontaneous resolution and low-fluence PDT. However, only in the PDT group, after disappearance of subretinal fluid, did it decrease to that of normal subjects.

Eye (2013) 27, 387-391; doi:10.1038/eye.2012.273; published online 4 January 2013

Keywords: choroidal thickness; enhanced depth imaging optical coherence tomography; lowfluence photodynamic therapy; spontaneously resolved CSC

\section{Introduction}

Central serous chorioretinopathy (CSC) is a common disorder, which is characterized by a serous neurosensory retinal or retinal pigment epithelium (RPE) detachment, involving the posterior pole. ${ }^{1,2}$ The episodes of acute CSC are generally self-limited with spontaneous resolution of detachment within 3-4 months, although the treatment with laser photocoagulation or photodynamic therapy (PDT) may be considered in the conditions, including recurrent or persistent CSC. ${ }^{3-5}$

Two theories in the pathogenesis of CSC have been proposed: one is choroidal dysfunction and the other is RPE dysfunction theory. ${ }^{5}$ Since Gass ${ }^{1}$ proposed that CSC originates in
Department of Ophthalmology, Ewha Womans University, School of Medicine, Mok-dong Hospital, Seoul, Republic of Korea

Correspondence: YT Kim, Department of Ophthalmology, Ewha

Womans University, School of Medicine, Mok-dong Hospital, 911-1, Mok-dong Yangcheon-ku, Seoul 158-710, Republic of Korea Tel: + 8222650 5154; Fax: + 82226504334 E-mail: jjongofhim@ hanmail.net.

Received: 30 April 2012 Accepted in revised form: 12 November 2012 Published online: 4 January 2013 
choriocapillaris hyperpermeability, several studies, based on indocyanine green angiography (ICGA) findings, demonstrated the evidences of choroidal vascular abnormalities in patients with CSC. ${ }^{4-9}$ Furthermore, recent studies evaluated the choroidal thickness in CSC using enhanced depth imaging optical coherence tomography (EDI-OCT), which can provide crosssectional images of choroid non-invasively and assess the effects of choroidal vascular hyperpermeability. ${ }^{10-16}$ Imamura et al ${ }^{11}$ reported that subfoveal choroidal thickness (SFCT), in eyes with CSC, was significantly larger than that of the normal eyes, as shown on EDIOCT. Previous studies showed that the two treatment modalities, PDT and focal laser photocoagulation, resulted in different changes on the choroidal thickness; EDI-OCT showed decrement of choroidal thickness after PDT, whereas it did not after laser photocoagulation. ${ }^{13,14}$

However, to our knowledge, there has been no report on the change of choroidal thickness in patients with spontaneously resolved CSC, using the EDI-OCT. Thus, this study aims to investigate the change of SFCT after spontaneous resolution of CSC and compared it with that of the after reduced fluence PDT for CSC. In addition, to estimate the SFCT, after complete resolution in two CSC groups, spontaneously resolved CSC and PDT-treated CSC, these were compared with that of the normal subjects.

\section{Methods}

Medical records of patients from December 2010 to March 2012 were reviewed. Patients were diagnosed by slit lamp biomicroscope and angiography-fluorescein angiography and ICGA. A total of 36 consecutive eyes of 36 patients with CSC were included. There were 16 patients presented with spontaneous resolution of CSC and 20 patients receiving ICGA-guided PDT with verteporfin for persistent subretinal fluid (SRF) of more than 3-month duration.

Best-corrected visual acuity (BCVA) measurement with Snellen chart and EDI spectral domain OCT (Spectralis, Heidelberg Engineering Inc., Heidelberg, Germany) were performed at each visit. BCVA was converted into log MAR (logarithm of minimal angle of resolution) scale for the purpose of the analysis.

Patients visited the outpatient clinic monthly for 2 months. After the first 2 months, patients with evidence of decreasing SRF were followed up monthly until complete absorption. In patients with evidence of persistent or increasing SRF, after 2 months of observation, PDT was employed for facilitating SRF absorption. To avoid the potential complications of conventional PDT, the patients had undergone a modified fluence PDT-reduced time of laser emission
(50 s) and total light energy $\left(30 \mathrm{~J} / \mathrm{cm}^{2}\right)$, using the standard dose of verteporfin $\left(6 \mathrm{mg} / \mathrm{m}^{2}\right)$ and laser intensity $\left(600 \mathrm{~mW} / \mathrm{cm}^{2}\right) .4,5,14,17-19$ The PDT spot size was determined by measuring the greatest linear dimension of the area of choroidal vascular hyperpermeability, corresponding to the origin of subfoveal fluid on ICGA. Patients receiving PDT were followed up and BCVA and SFCT using EDI-OCT at 7 day and 1 month after treatment were evaluated.

Patients with history of other retinochoroidal diseases were excluded, as well as patients who had undergone any other vitreoretinal interventions: laser photocoagulation, intravitreal injection or vitreoretinal surgery. Patients with high myopia (Spherical equivalent $<-6.0 \mathrm{D})$ or patients with history of refractive surgery were not included. We also excluded patients taking medication implicated in altering the choroidal vasculature, including corticosteroid and sildenafil. ${ }^{20,21}$ For the estimation of SFCT, after complete resolution of SRF in CSC, SFCT of 32 normal volunteers were also evaluated.

To obtain the images of choroid, we adopted EDI mode, which was provided by the manufacturer (Heidelberg Engineering). For the macula going through the foveola, $9 \mathrm{~mm}$ horizontal and vertical sections, which contained 100 duplicated scans, were obtained. A single masked observer measured the choroidal thickness, manually, from a vertical and horizontal image, using the contained Heidelberg eye explorer software (version 1.5.12.0; Heidelberg Engineering Inc.) and averaged the two measurements. The SFCT was defined as the vertical distance from the hyper-reflective line of the Bruch's membrane to innermost hyperreflective line of chorioscleral interface, under the center of the fovea (Figure 1). ${ }^{10}$

SPSS software version 18.0 for Windows (SPSS Inc., Chicago, IL, USA) was used for statistical analysis. The changes in the measurements of the choroidal thickness and BCVA, after resolution of CSC in the observation and PDT groups, were analyzed using the Wilcoxon's signedrank test, respectively. For the comparisons of the SFCT of the eyes with CSC with that of the normal eyes, MannWhitney $U$-test with Bonferroni correction was also employed. Results were considered to be statistically significant at $P$-values $<0.05$.

\section{Results}

Clinical characteristics of normal subjects, patients with spontaneously resolved CSC and patients who underwent PDT are summarized in Table 1. There was no significant difference in the mean age, gender, refractive error and systemic hypertension among the three groups. 

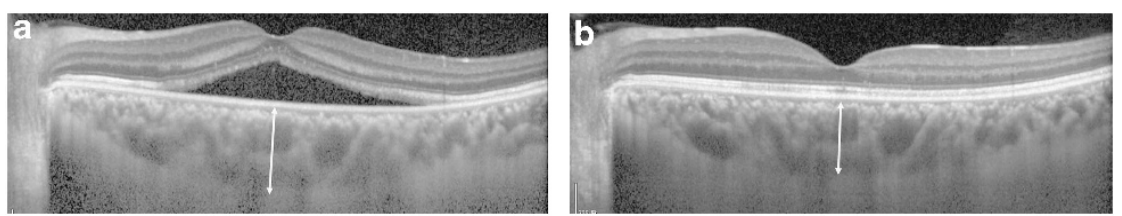

Figure 1 Representative enhanced depth imaging OCT images of a patient who showed spontaneous resolution of CSC. Subfoveal choroidal thickness was $475 \mu \mathrm{m}$ at first visit (a), and decreased to $410 \mu \mathrm{m}$ after complete absorption of SRF (b). The arrows indicate the subfoveal choroidal thickness, the vertical distance from hyper-reflective line of the RPE Bruch's membrane to innermost hyperreflective line of chorioscleral interface.

Table 1 Demographic characteristics of unilateral central chorioretinopathy patients with and without treatment, and normal subjects

\begin{tabular}{|c|c|c|c|c|}
\hline & \multirow{2}{*}{$\begin{array}{l}\text { Normal } \\
\text { subjects }\end{array}$} & \multicolumn{2}{|c|}{$\begin{array}{l}\text { Patients } \\
\text { with CSC }\end{array}$} & \multirow{2}{*}{$\begin{array}{l}\mathrm{P}- \\
\text { value }\end{array}$} \\
\hline & & Observation & $\begin{array}{l}\text { Low-fluence } \\
\text { PDT }\end{array}$ & \\
\hline $\begin{array}{l}\text { No. of eyes } \\
\text { Age, mean } \pm S D \\
\text { (year) }\end{array}$ & $\begin{array}{c}32 \\
45.00 \pm 13.51\end{array}$ & $\begin{array}{c}16 \\
47.25 \pm 7.66\end{array}$ & $\begin{array}{c}20 \\
48.90 \pm 11.18\end{array}$ & $0.609^{a}$ \\
\hline $\begin{array}{l}\text { Sex, female:male } \\
\text { SE, mean } \pm \text { SD (D) } \\
\text { Systemic } \\
\text { HTN, } n(\%)\end{array}$ & $\begin{array}{c}13: 19 \\
-1.14 \pm 2.05 \\
5(15.6 \%)\end{array}$ & $\begin{array}{c}6: 10 \\
-0.33 \pm 1.35 \\
2(12.5 \%)\end{array}$ & $\begin{array}{c}3: 17 \\
-0.72 \pm 0.76 \\
7(35.0 \%)\end{array}$ & $\begin{array}{l}0.139^{\mathrm{b}} \\
0.337^{\mathrm{a}} \\
0.160^{\mathrm{b}}\end{array}$ \\
\hline
\end{tabular}

Abbreviations: CSC, central serous chorioretinopathy; PDT, photodynamic therapy; SE, spherical equivalent; HTN, hypertension.

${ }^{a}$ Kruskal-Wallis test.

${ }^{\mathrm{b}} \chi^{2}$ test.

The mean baseline visual acuity and SFCT are shown in Table 2. At the baseline, the SFCT in the two CSC groups was greater than that in normal subjects, which was $295.17 \pm 73.31 \mu \mathrm{m}$. No significant differences in the baseline visual acuity and SFCT between both CSC groups were detected.

In spontaneously resolved CSC, the SFCT significantly decreased from $459.16 \pm 77.50 \mu \mathrm{m}$ at the baseline to $419.31 \pm 54.49 \mu \mathrm{m}$ after complete absorption of SRF $(P=0.015$; Figure 1$)$ in line with improvement of log MAR BCVA from $0.54 \pm 0.47$ to $0.08 \pm 0.13(P=0.001)$. The PDT group, at 1 month after treatment, also showed notable reduction in the SFCT from $416.43 \pm 74.01$ to $349.50 \pm 88.99 \mu \mathrm{m}$ with reattachment of the retina $(P<0.001)$.

The SFCT after absorption of SRF without any intervention significantly increased compared with that of the normal subjects $(P<0.001)$. On the other hand, there was no statistically significant difference in SFCT between the low-fluence PDT-treated patients and normal subjects $(P=0.087)$.

\section{Discussion}

In prior studies, the characteristic ICGA findings in patients with CSC were demonstrated as follows: (1)
Table 2 Changes of subfoveal choroidal thickness and visual acuity of the affected eyes

\begin{tabular}{lccc}
\hline & Baseline & After resolution & P-value $^{\mathrm{a}}$ \\
\hline Observation & & & \\
SFCT $(\mu \mathrm{m})$ (range) & $459.16 \pm 77.50$ & $419.31 \pm 54.49$ & 0.015 \\
& $(363.50-687.00)$ & $(330.50-489.00)$ & \\
Log MAR BCVA & $0.54 \pm 0.47$ & $0.08 \pm 0.13$ & 0.001 \\
& & & \\
Low-fluence PDT & & & \\
SFCT $(\mu$ m) (range) & $416.43 \pm 74.01$ & $349.50 \pm 88.99$ & 0.000 \\
Log MAR BCVA & $(306.50-550.00)$ & $(159.00-466.50)$ & \\
& $0.32 \pm 0.30$ & $0.22 \pm 0.22$ & 0.010 \\
\hline
\end{tabular}

Abbreviations: PDT, photodynamic therapy; SFCT, subfoveal choroidal thickness; log MAR BCVA, logarithm of minimal angle of resolution bestcorrected visual acuity.

All data are presented as mean \pm SD.

${ }^{\text {a} W i l c o x o n ' s ~ s i g n e d-r a n k ~ t e s t . ~}$

choriocapillaris hyperpermeability; (2) delayed arterial filling; and (3) venous dilation or congestion. ${ }^{4-9}$ The author also found these choroidal vascular abnormalities in a previous study. ${ }^{15}$ ICGA showed choroidal vascular hyperpermeability in $28(93.3 \%)$ eyes with CSC and in 23 (73.3\%) unaffected fellow eyes. ${ }^{15}$ Choroidal vascular dilation was detected in $21(70.0 \%)$ eyes with CSC and in $18(60.0 \%)$ unaffected fellow eyes. ${ }^{15}$

These disturbances of choroidal circulation in patients with CSC give rise to increase the hydrostatic pressure in choroid, and subsequently, to increase the thickness of choroid. ${ }^{11}$ EDI-OCT, which enabled apparent visualization of choroidal vasculature, showed increased subfoveal thickness in eyes with CSC. ${ }^{11-16}$

This study showed that the thickness of choroid in the eyes with CSC decreased after spontaneous disappearance of SRF; however, not to those of the normal level. This result corresponds to the previous study, which demonstrated the persistent choroidal vascular abnormalities on ICGA, even after the resolution of active leakage from the level of RPE. ${ }^{7}$ According to these reports using the EDI-OCT and ICGA, the absorption of SRF might certainly precede the normalization of subfoveal choroidal vasculopathy in spontaneous resolution of CSC. This finding does not mean that the change of choroidal vasculature follows 
the absorption of SRF. It remains unclear at first; the natural resolution of SRF results from either decreased effusion from choroid or healing of the RPE microrip, or possibly both, as suggested by the report by Gupta et al. ${ }^{22}$

Nevertheless, considering the fact that the SFCT after resolution decreased from the baseline regardless of treatment, the decrement of choroidal thickness might be an essential prerequisite or concomitant phenomenon for healing of CSC.

The SFCT after a spontaneous resolution might also indicate that increased choroidal thickness is a feature of individuals, who are apt to develop CSC. Authors and other investigators reported that the choroid was thickened in both eyes of those patients with unilateral involvement of CSC. ${ }^{11,15}$ On the other hand, increased choroidal thickness may suggest the preclinical phase of CSC, which constitutes the environment for active CSC. However, these cannot be elucidated from this study because of its short follow-up duration.

As stated previously, the modified PDT protocol used in this study was different from the widespread low-fluence PDT protocol.4,5,17-19 Generally, in lowfluence PDT, a laser light at $689 \mathrm{~nm}$ delivered $25 \mathrm{~J} / \mathrm{cm}^{2}$ with an intensity of $300 \mathrm{~mW} / \mathrm{cm}^{2}$ over $83 \mathrm{~s} .{ }^{17,19}$ Whereas, in our study, the same wavelength laser with $600 \mathrm{~mW} /$ $\mathrm{cm}^{2}$ intensity as the full dose PDT was applied for $50 \mathrm{~s}$ and total light energy $\left(30 \mathrm{~J} / \mathrm{cm}^{2}\right)$. However, in spite of little discrepancy in the protocol, the success rate (about $83.3 \%$ ) at 1 month on CSC is comparable with the previous outcomes (75-96\%) after PDT. ${ }^{13,14,17-19,23}$

Our results of decreased choroidal thickness following PDT concur with prior reports. ${ }^{13,14,16}$ It resulted from the action mechanism of PDT. That is, PDT resulted in choroidal vascular hypoperfusion and reduction of the choroidal exudation in the areas of SRF production, presented as the decrement of SFCT on EDIOCT. 4,5,13,14,16 It was reinforced by the reports of Maruko et al ${ }^{13,14}$ that the choroidal thickness was reduced after ICGA-guided PDT, whereas it was not after laser photocoagulation. ${ }^{13,14}$

In the recent study, the choroidal thickness, following a low-fluence PDT, was nearly as thin as that of the normal control group, unlike the natural resolution of CSC. This finding suggested that the healing mechanism of PDT might be different from that of the spontaneous resolution. In the PDT group, reattachment of retinal detachment might occur subsequent to the decrement of choroidal thickness, and finally, be accompanied by the normalization of SFCT. The discrepancy may also explain the reasons why the recurrence rate of CSC was relatively lower after PDT, which induced short-term occlusion of choriocapillary and long-term choroidal vascular remodeling. ${ }^{13,14,17,18}$
The main limitation of this study is that the follow-up was relatively short. Because the visual impairment from CSC is not severe in general, patients did not visit the clinic after the disappearance of the symptoms. Thus, although longitudinal observation would be helpful, it was impossible.

The manual measurement of choroidal thickness, by a single observer regardless of the diurnal variation, is another drawback of this study. ${ }^{24}$ However, authors thought that averaging the choroidal thickness in two different sections-vertical and horizontal sectionswould decrease the measuring error. Previous studies also showed that the measurements of choroidal thickness, using EDI-OCT, have good reproducibility and repeatability. ${ }^{25,26}$ Besides, considering the similarity of the normal values of this study and that of the previous studies, measuring error may not be significant. ${ }^{10,26,27}$

In conclusion, increased SFCT of patients with CSC was reduced after spontaneous resolution and lowfluence PDT. While it did not decrease to that of the normal subjects in spontaneously resolved patients, the choroidal thickness in PDT received patients was reduced to its normal level. These results showed that the CSC was resolved in a different process and mechanism, even though the decrement of choroidal vascularity appeared during the recovery in both groups.

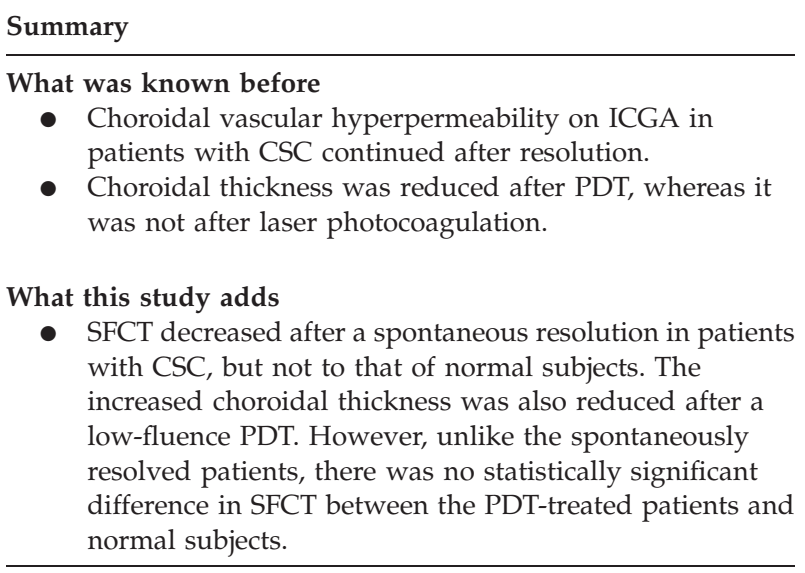

\section{Conflict of interest}

The authors declare no conflict of interest.

\section{References}

1 Gass JD. Pathogenesis of disciform detachment of the neuroepithelium. II. Idiopathic central serous chorioretinopathy. Am J Ophthalmol 1967; 63(3, Suppl): 1-139.

2 Klais CM, Ober MD, Ciardella AP, Yannuzzi LA. Central serous chorioretinopathy. In: Ryan SJ (eds) Retina. Vol 2 Elsevier Mosby: Philadelphia 2006) pp 1135-1161.

3 Yannuzzi LA. Type-A behavior and central serous chorioretinopathy. Retina 1987; 7(2): 111-131. 
4 Ross A, Ross AH, Mohamed Q. Review and update of central serous chorioretinopathy. Curr Opin Ophthalmol 2011; 22(3): 166-173.

5 Gemenetzi M, De Salvo G, Lotery AJ. Central serous chorioretinopathy: an update on pathogenesis and treatment. Eye 2010; 24(12): 1743-1756.

6 Piccolino FC, Borgia L. Central serous chorioretinopathy and indocyanine green angiography. Retina 1994; 14(3): 231-242.

7 Iida T, Kishi S, Hagimura N, Shimizu K. Persistent and bilateral choroidal vascular abnormalities in central serous chorioretinopathy. Retina 1999; 19(6): 508-512.

8 Scheider A, Nasemann JE, Lund OE. Fluorescein and indocyanine green angiographies of central serous chorioretinopathy by scanning laser ophthalmoscopy. Am J Ophthalmol 1993; 115(1): 50-56.

9 Prünte C, Flammer J. Choroidal capillary and venous congestion in central serous chorioretinopathy. Am J Ophthalmol 1996; 121(1): 26-34.

10 Spaide RF, Koizumi H, Pozzoni MC. Enhanced depth imaging spectral-domain optical coherence tomography. Am J Ophthalmol 2008; 146(4): 496-500.

11 Imamura Y, Fujiwara T, Margolis R, Spaide RF. Enhanced depth imaging optical coherence tomography of the choroid in central serous chorioretinopathy. Retina 2009; 29(10): 1469-1473.

12 Maruko I, Iida T, Sugano Y, Ojima A, Sekiryu T. Subfoveal choroidal thickness in fellow eyes of patients with central serous chorioretinopathy. Retina 2011; 31(8): 1603-1608.

13 Maruko I, Iida T, Sugano Y, Ojima A, Ogasawara M, Spaide RF. Subfoveal choroidal thickness after treatment of central serous chorioretinopathy. Ophthalmology 2010; 117(9): 1792-1799.

14 Maruko I, Iida T, Sugano Y, Furuta M, Sekiryu T. One-year choroidal thickness results after photodynamic therapy for central serous chorioretinopathy. Retina 2011; 31(9): 1921-1927.

15 Kim YT, Kang SW, Bai KH. Choroidal thickness in both eyes of patients with unilaterally active central serous chorioretinopathy. Eye 2011; 25(12): 1635-1640.

16 Wirostko WJ, Nordgren RN, Dubis AM. Topographical choroidal thickness change following PDT for CSC: an OCT case report. J Ophthalmol 2012; 2012: 347206

17 Smretschnig E, Ansari-shahrezaei S, Moussa S, Glittenberg C, Krebs I, Binder S. Half-fluence photodynamic therapy in acute central serous chorioretinopathy.. Retina 2012; 32(10): 2014-2019.

18 Chan WM, Lai TY, Lai RY, Liu DT, Lam DS. Half-dose verteporfin photodynamic therapy for acute central serous chorioretinopathy; one-year results of a randomized controlled trial. Ophthalomolgy 2008; 115(10): 1756-1765.

19 Reibaldi M, Cardascia N, Longo A, Furino C, Avitabile T, Faro $\mathrm{S}$ et al. Standard-fluence versus low-fluence photodynamic therapy in chronic central serous chorioretinopathy: A nonrandomized clinical trial. Am J Ophthalmol 2010; 149(2): 307-315.

20 Jampol LM, Weinreb R, Yannuzzi L. Involvement of corticosteroids and catecholamines in the pathogenesis of central serous chorioretinopathy: A rationale for new treatment strategies. Ophthalmology 2002; 109(10): 1834-1837.

21 Vance SK, Imamura Y, Freund KB. The effects of sildenafil citrate on choroidal thickness as determined by enhanced depth imaging optical coherence tomography. Retina 2011; 31(2): 332-335

22 Gupta V, Gupta P, Dogra MR, Gupta A. Spontaneous closure of retinal pigment epithelium microrip in the natural course of central serous chorioretinopathy. Eye 2010; 24(4): 595-599.

23 Cardillo Piccolino F, Eandi CM, Ventre L, Rigault de la Longrais RC, Grignolo FM. Photodynamic therapy for chronic central serous chorioretinopathy. Retina 2003; 23(6): 752-763.

24 Brown JS, Flitcroft DI, Ying GS, Francis EL, Schmid GF, Quinn GE et al. In vivo human choroidal thickness measurements: evidence for diurnal fluctuations. Invest Ophthalmol Vis Sci 2009; 50(1): 5-12.

25 Yamashita T, Yamashita T, Shirasawa M, Arimura N, Terasaki H, Sakamoto T. Repeatability and reproducibility of subfoveal choroidal thickness in normal eyes of Japanese using different SD-OCT devices. Invest Ophthalmol Vis Sci 2012; 53(3): 1102-1107.

26 Branchini L, Regatieri CV, Flores-Moreno I, Baumann B, Fujimoto JG, Duker JS. Reproducibility of choroidal thickness measurements across three spectral domain optical coherence tomography systems. Ophthalmology 2012; 119(1): 119-123.

27 Margolis R, Spaide RF. A pilot study of enhanced depth imaging optical coherence tomography of the choroid in normal eyes. Am J Ophthalmol 2009; 147(5): 811-815. 